\title{
Mueller-matrix differential diagnosis of biological crystallites phase anisotropy
}

\author{
V.O. Ushenko, G.D. Koval, V.T. Bachinskiy, L.Ya. Kushnerick, M. Garazdiyk, \\ M. M. Dominikov, O.V. Dronenko \\ Optics and Publishing Department, \\ Chernivtsi National University, \\ 2 Kotsyubinsky Str., Chernivtsi, 58012, Ukraine \\ alexander.dubolazov@gmail.com
}

\begin{abstract}
The information is given about the optical arrangement, in which the elements of the coordinate allocations of the Mueller-matrix of the optically thin polycrystalline layers are defined.

The algorisms are given and the experimental methods of measuring the Jones-matrix pictures are analyzed.

The experimental results of the investigation of the statistical, correlative and fractal parameters are represented and they characterize the Mueller-matrix pictures of polycrystalline systems of the essential types of human's amino acid.
\end{abstract}

Keywords: polarimetry, Mueller-matrix, birefringence, biological tissues.

\section{Introduction}

The scattering of the optical radiation by biological objects and environments is in approximation of statistical averaging of photometric and polarized parameters. The most widespread methods of diagnostics are those, which are based on the study of the scattered radiation fields by means of the classical photometry [1,2], Stokes-polarimetry and Mueller-matrix optics [3,4]. Simultaneously with these research areas laser methods of investigation of optical-heterogeneous biological structures have been developing. These methods are correlative optics and speckles' optics [5], which use coherent polarized radiation as a probe.

The coherence of laser rays stipulated the necessity of elaboration of other approaches to the analysis of the scattered radiation fields - fractal optics [6], singular optics [7,8]. On their basis it was defined the direct interdependence between the set of the statistical moments of the 1-st and 2-D sequences, functions of autocorrelation and fractal dimension, which describe optical anisotropic structure of biological tissues and their laser pictures by polarized parameters ( the coordinate distribution of azimuths and ellipticity of polarization, the Mueller-matrix elements) [9].

From other point of view there is a wide range of less studied biological objects of another type. First of all different liquids such as: blood, gall, synovial fluid, cerebrospinal fluid (liquor) and others that belong to them. That is why it is urgent to continue the development of new approaches to the analysis of the vector structure of the laser radiation fields, transformed not only by the optical anisotropic layers of biological tissues, but also by the membranes of biological fluids, especially by the polycrystalline systems of their proteins.

The aim of the work was development and grounding new approaches to the classification and differentiation of the polarized properties of the polycrystalline systems of biological layers by means of improvement of the Mueller-matrix mapping methods and elaboration of statistical correlative and fractal approaches to their analysis.

2. Optical scheme of Mueller-matrix mapping of optical-anisotropic biological layers

There is an optical scheme of polarimeter for the measuring the totality of the coordinate distribution of the Mueller-matrix elements of biological fluids smears at the picture 1 [10].

Eleventh International Conference on Correlation Optics, edited by Oleg V. Angelsky, Proc. of SPIE Vol. 9066, 906621 - @ 2013 SPIE · CCC code: 0277-786X/13/\$18 - doi: 10.1117/12.2053863 


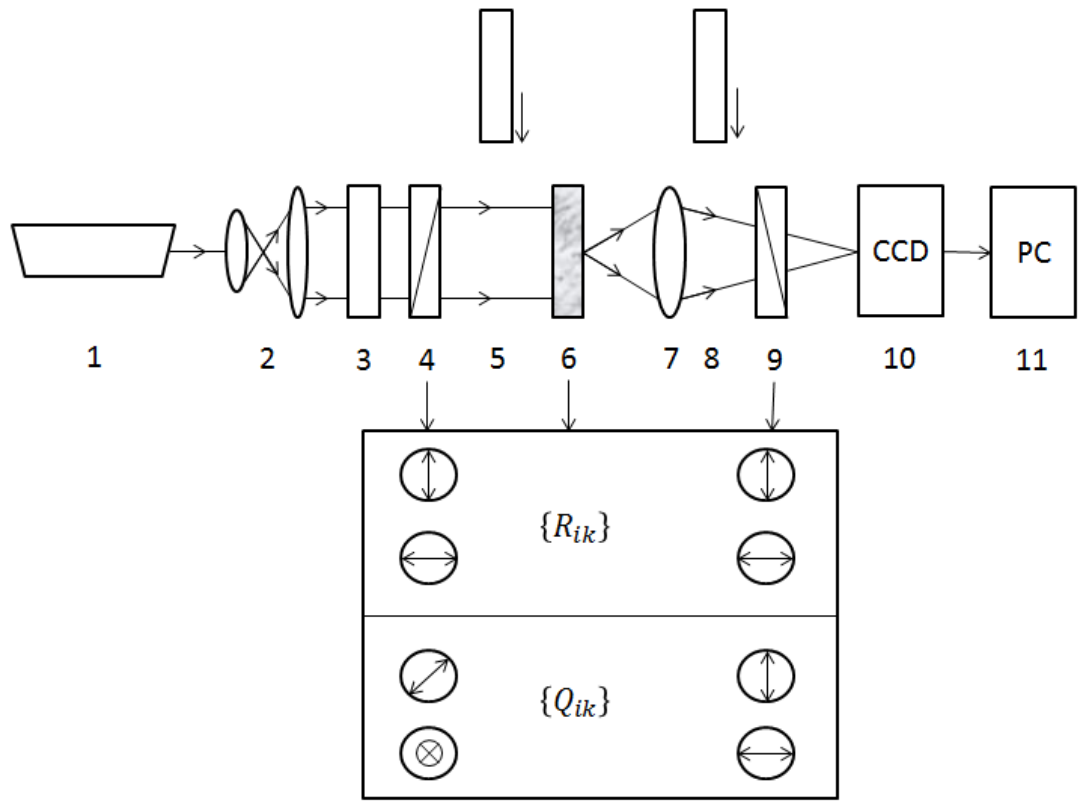

Figure 1. The optical scheme of polarimeter, where 1 - a He-Ne laser, 2 - a collimator, 3 - a fixed quarter-wave plate; 5,8-a mechanically movable quarter-wave plate; 4,8 - a polarizer and an analyzer; 6 - an object of study; 7 - an object glass; 10 - CCD camera; 11 - a computer.

The illumination was conducted by the parallel $\left(\varnothing=10^{4} \mathrm{mkm}\right)$ bundle of rays of the He-Ne laser $\quad(\lambda=$ $0.6328 \mathrm{mkm}, \mathrm{W}=5.0 \mathrm{~mW}$ ). A polarized illuminant consists of the quarter-wave plates $3 ; 5$ and the polarizer 4 , that provide the formation of laser cluster with arbitrary azimuth $0^{\circ} \leq \alpha_{0} \leq 180^{\circ}$ of with the polarizational ellipticity $0^{\circ} \leq \beta_{0} \leq 90^{\circ}$.

Polarizable images of biological layers were projected in the actinic plane $(800 \times 600$ pixels $)$ of the CCDcamera 10 with the help of microlens. The camera provided measure range of the image of structural elemens biological liquids for the following size $2 \mathrm{mcm}-2000 \mathrm{mcm}$.

The conditions of the experiment were chosen so that to eliminate spatio-angular aperture filtration in case of biological layers formation. It was provided by coordination of angular characteristics of the indicatrixes of the light diffusion with the biological liquid samples $\left(\Omega \approx 16^{\circ}\right)$ and angular aperture of microlens $\left(\Delta \omega=20^{\circ}\right)$. Here $\Omega$ - angular cone of the indicatrixes in which $98 \%$ of the whole scattered radiation energy are concentrated. plate 8 .

Image analysis of the biological liquids' smears was done with the help of polarizer 9 and quarterwave

\section{Mueller-matrix images of the polycrystal networks of the optic-thin amino acid layers}

In order to get objective criteria of the polarizable manifestations of the optic anisotropy of different polycrystal network types we carried out complex investigation of the coordinate allocations of the Muller's matrix elements $\kappa_{11}(\mathrm{~m} \times \mathrm{n})$ and $\kappa_{12 ; 21}(\mathrm{~m} \times \mathrm{n})$ which characterize chiefly the orientational $\rho$ and phasal $\delta$ structure manifestations of the group of liquid crystal amino acids [6-19].

On the series fig. 2- fig. 5 are shown the series of the experimental measured Muller-matrix images of the "orientational" $\mathrm{N}_{11}(\mathrm{~m} \times \mathrm{n})$ and "phasal" $\mathrm{N}_{12 ; 21}(\mathrm{~m} \times \mathrm{n})$ elements and three-dimensional reconstructions (histogram) of their value $H\left(\aleph_{11}\right), H\left(\aleph_{12 ; 21}\right)$, autocorrelative functions $G_{11}(\Delta \chi) ; G_{12 ; 21}(\Delta \chi)$, logarithmic spectral dependencies $\operatorname{LogPSD}\left(\aleph_{11}\right) ; \log P S D\left(\aleph_{12 ; 21}\right)$ of the coordinate allocations of values of matrix elements of polycrystal layers of the basic types of methionine amino acids (fig.2 and fig.3) and proline amino acids (fig.4 and fig.5) respectively. 


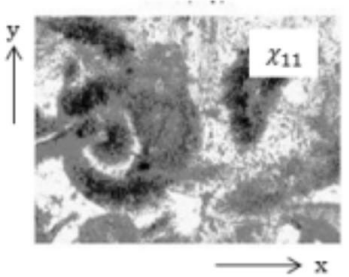

(a)

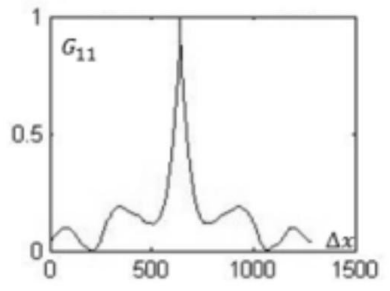

(c)

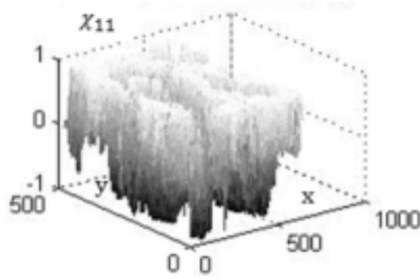

(b)

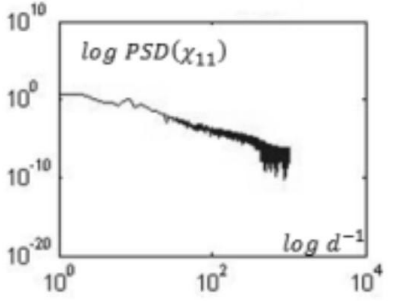

(d)

Figure. 2. Coordinative $\aleph_{11}(\mathrm{~m} \times \mathrm{n})$, stochastic $H\left(\aleph_{11}\right)$, correlative $G_{11}(\Delta m, \Delta n)$ and selfsimilar $\log P S D\left(\aleph_{11}\right)$ structure of the Muller's matrix $\aleph_{11}$ element of the polycrystal network of methionine layer.

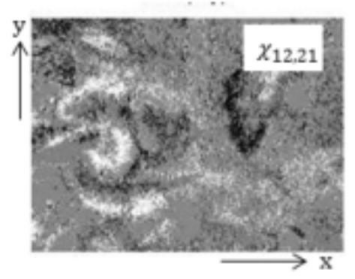

(a)

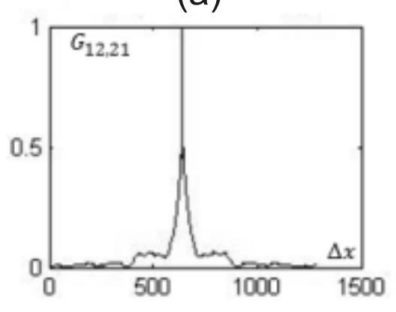

(c)

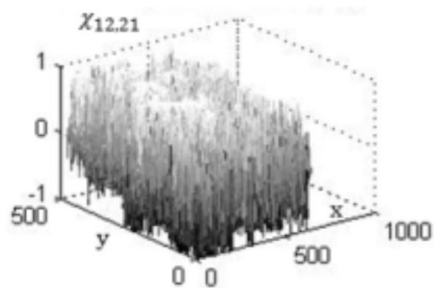

(b)

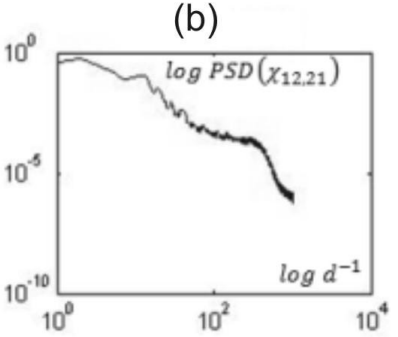

(d)

Figure. 3. Coordinative $\aleph_{12 ; 21}(\mathrm{~m} \times \mathrm{n})$, stochastic $H\left(\aleph_{12 ; 21}\right), G_{12 ; 21}(\Delta m, \Delta n)$ and selfsimilar $\log P S D\left(\aleph_{12 ; 21}\right)$ structure of the Muller's matrix $\aleph_{12 ; 21}$ element of the polycrystal network of methionine layer. 


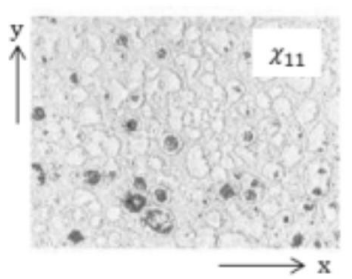

(a)

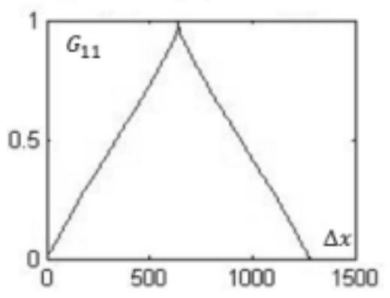

(c)

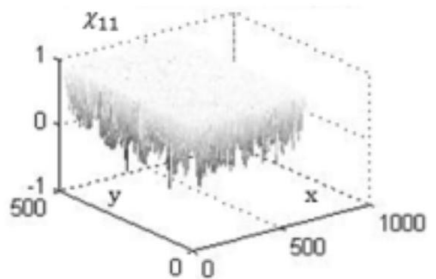

(b)

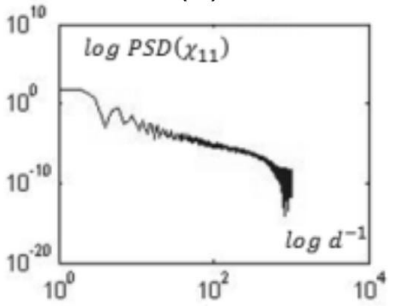

(d)

Figure. 4. Coordinative $\mathrm{N}_{11}(\mathrm{~m} \times \mathrm{n})$, stochastic $H\left(\mathrm{\kappa}_{11}\right)$, correlative $G_{11}(\Delta m, \Delta n)$ and selfsimilar $\log P S D\left(\mathrm{\kappa}_{11}\right)$ structure of the Muller's matrix $\kappa_{11}$ element of the polycrystal network of proline layer.

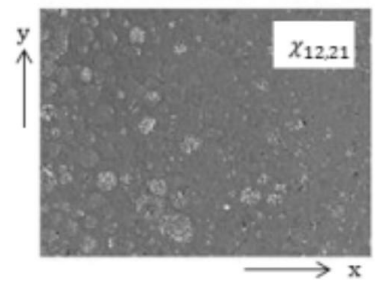

(a)

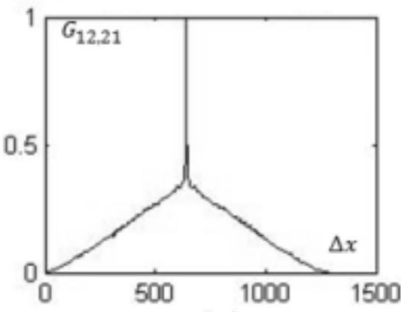

(c)

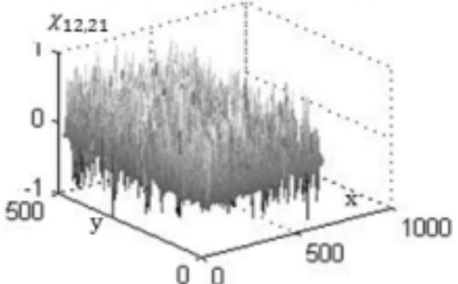

(b)

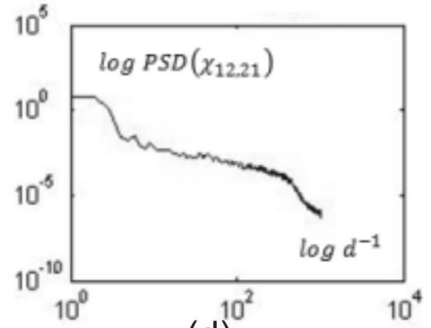

(d)

Figure. 5. Coordinative $\aleph_{12 ; 21}(\mathrm{~m} \times \mathrm{n})$, stochastic $H\left(\aleph_{12 ; 21}\right), G_{12 ; 21}(\Delta m, \Delta n)$ and selfsimilar $\log P S D\left(\aleph_{12 ; 21}\right)$ structure of the Muller's matrix $\aleph_{12 ; 21}$ element of the polycrystal network of proline layer.

The obtained results of the investigation of Muller-matrix images of "orientational" $\times 11(\mathrm{~m} \times \mathrm{n})$ and "phasal" $\kappa_{12 ; 21}(\mathrm{~m} \times \mathrm{n})$ elements of polycrystal layers of the basic human amino acid types have shown [6-12]:

The features of orientational structure lines of optical networks of partial amino acid crystals make significant influence. This is indicated by a wide range of variation $0 \leq \Delta \aleph_{11} \leq 1$ of eigenvalues of the matrix element $\aleph_{11}(m \times n)$ of the crystal layers major types of amino acids (Fig.2, Fig.4 fragments(a)). Besides all the coordinate distributions of " orientational "Mueller matrix elements are individual for polycrystalline networks with different geometric constructions (Fig. 2, Fig. 4 fragments ( b)). 2. Differences between the coordinate distributions (histograms) "phase" $\aleph_{12 ; 21}(m \times n)$ element (Fig. 4, Fig. 5 fragments (a)) of polycrystalline networks of different biochemical composition of amino acids are not as distinct (Fig. 4, Fig. 5 fragments (b)) as for Muller - matrix image " orientational " items. This similarity in our opinion is related to the parameter values close double ray refraction of glycine amino acids, methionine and proline. 
3. Autocorrelation functions $G_{11: 12 ; 21}(\Delta x)$ of coordinate distributions of Mueller matrix elements of the crystal layers of amino acids with distinct dendritic and spherelithic geometry are declining dependence with strongly pronounced fluctuation of proper values (Fig. 2 and Fig. 4 fragments (c)) . 4. Sets of values of "orientational" $\aleph_{11}(m \times n)$ Mueller matrix elements are almost fractal, and for "phase" matrix element $\aleph_{12 ; 21}(m \times n)$ multifractal. The corresponding logarithmic dependencies $\log P S D\left(\aleph_{11}\right)$ are characterized by continuous range of variation of all geometric dimensions of partial crystal angle (Fig. 2, Fig. 4 fragments (d ) ).

For dependencies $\log P S D\left(\aleph_{12: 21}\right)$ approximating curves with two angles are characteristic

(Fig. 5

fragments (d )). In our opinion, this fact can be related to multiple scale and coordinate orderly change of orientation of the optical axes of the partial amino acid crystals with simultaneous multiple phase change period $\delta$.

The results of quantitative analysis of values and range of variation statistics, correlation and spectral moments [5] that characterize coordinate distributions $\aleph_{i k}(m \times n)$ of Mueller matrix elements polycrystalline layers major types of amino acids are given in Table 1.

Table 1. Statistical ( $M, \sigma, A, E)$, correlation $\left(K_{i=1 ; 2 ; 3 ; 4}\right)$, spectral $\left(S_{i=1 ; 2 ; 3 ; 4}\right)$ parameters of Mueller - matrix images $\aleph_{i k}(m \times n)$ polycrystalline layers amino acids.

\begin{tabular}{|c|c|c|c|c|c|c|c|c|c|c|c|}
\hline \multicolumn{6}{|c|}{$\aleph_{11}(m \times n)$} & \multicolumn{6}{|c|}{$\aleph_{12 ; 21}(m \times n)$} \\
\hline \multicolumn{12}{|c|}{ Metionin } \\
\hline$M$ & 0,31 & $K_{1}$ & 0,52 & $S_{1}$ & 0,48 & $M$ & 0,51 & $K_{1}$ & 0,51 & $S_{1}$ & 0,5 \\
\hline$\sigma$ & 0,15 & $K_{2}$ & 0,11 & $S_{2}$ & 0,13 & $\sigma$ & 0,24 & $K_{2}$ & 0,11 & $S_{2}$ & 0,11 \\
\hline$A$ & 0,53 & $K_{3}$ & 0,57 & $S_{3}$ & 0,47 & $A$ & 0,18 & $K_{3}$ & 0,24 & $S_{3}$ & 0,31 \\
\hline$E$ & 0,68 & $K_{4}$ & 2,12 & $S_{4}$ & 0,39 & $E$ & 0,12 & $K_{4}$ & 0,91 & $S_{4}$ & 0,27 \\
\hline \multicolumn{12}{|c|}{ Prolin } \\
\hline$M$ & 0,39 & $K_{1}$ & 0,45 & $S_{1}$ & 0,56 & $M$ & 0,48 & $K_{1}$ & 0,48 & $S_{1}$ & 0,52 \\
\hline$\sigma$ & 0,28 & $K_{2}$ & 0,1 & $S_{2}$ & 0,13 & $\sigma$ & 0,32 & $K_{2}$ & 0,07 & $S_{2}$ & 0,09 \\
\hline$A$ & 0,12 & $K_{3}$ & 0,24 & $S_{3}$ & 0,48 & $A$ & 0,12 & $K_{3}$ & 0,36 & $S_{3}$ & 0,21 \\
\hline$E$ & 0,09 & $K_{4}$ & 1,38 & $S_{4}$ & 0,27 & $E$ & 0,09 & $K_{4}$ & 1,15 & $S_{4}$ & 0,18 \\
\hline
\end{tabular}

\section{Conclusions}

1. The entire set of statistics, correlation and spectral moments 1 st -4 th orders that characterize coordinate distributions of Mueller matrix elements has individual sets of values, depending on the fiber - geometrical parameters of polycrystalline chains of amino acids.

2. There exists a satisfactory correlation between the computer simulation and experimental studies of the structure Muller - matrix images.

3. Disordering directions of optical axes of the partial amino acid crystals are displayed in the following changes of quantitative parameters - a decrease of the statistical moments of the 3rd and 4th order coordinate the distribution of "orientational" Mueller matrix element according to the following geometry polycrystalline networks "dendritic net - sferolitna Network - an ensemble of clusters '; damping oscillations autocorrelation functions Muller - matrix images and reducing the 
relative values of the asymmetry $\left(K_{3}\right)$ and excess $\left(K_{4}\right)$ of such dependencies, increasing the spectral moments of the 3rd and 4th order, describing logarithmic dependence of the power spectrum of coordinate distribution of " orientation " Mueller matrix elements .

4. The range of differences between the values of statistical, correlation and spectral moments 1st - 4th order, describing the distribution of values "phase" elements of the Mueller matrix of polycrystalline networks of different types of amino acids "dendritic net - spherelithic Network cluster ensemble", 2 - 3 times smaller than in the case of similar quantitative parameters for the "orientational" Muller - matrix images.

\section{REFERENCES}

[1] Bueno, J.M. Spatially resolved polarization properties for in vitro corneas / J.M. Bueno, J. Jaronski // Ophthal. Physiol. Opt. Vol. 21, No. 5, 384-392 (2001).

[2] Bueno, J.M. Measurements of the corneal birefringence with a liquid-crystal imaging polariscope / J.M. Bueno, F. Vargas-Martin // Applied Optics, Vol. 41, No. 1, 116-124 (January 2002)

[3] Bueno, J.M. Polarization properties of the in vitro old human crystalline lens / J.M. Bueno, M.C.W. Campbell // Ophthal. Physiol. Opt. 23, 109-118 (2003)

[4] Tower, T.T. Alignment Maps of Tissues: I. Microscopic Elliptical Polarimetry / T.T.Tower, R.T.Tranquillo // Biophys. J., Vol. 81, 2954-2963, 2001.

[5] Tower, T.T. Alignment Maps of Tissues: II. Fast Harmonic Analysis for Imaging / T.T.Tower, R.T.Tranquillo // Biophys. J., Vol. 81, 2964-2971, 2001.

[6] Shribak, Techniques for fast and sensitive measurements of two-dimensional birefringence distributions / Shribak, R. Oldenbourg // Appl. Opt., Vol. 42, 3009-3017, 2003.

[7] Smith, M.H. Mueller matrix imaging polarimetry in dermatology / M.H. Smith, P. Burke, A. Lompado, E. Tanner, L.W. Hillman // Proc. SPIE, Vol.3991, 210-216 (2000).

[8] Angelsky, O.,V., Burkovets, D.,N., Kovalchuk, A.,V., Hanson, S., G., "Fractal description of rough surfaces," Applied optics 41 (22), 4620-4629 (2002).

[9] Angelsky O.,V., Ushenko A., G., Ushenko Y., A., Ushenko Y., G., "Polarization singularities of the object field of skin surface," Journal of Physics D: Applied Physics 39 (16), 3547 (2006).

[10] Angelsky, O., V., Burkovets, D., N., Kovalchuk, A., V., Hanson, S., G., "Fractal description of rough surfaces," Applied optics 41 (22), 4620-4629 (2002).

[11] Angelsky, O., V., Ushenko, A.,G., Burkovets, D., N., Ushenko, Y., A., "Polarization visualization and selection of biotissue image two-layer scattering medium," Journal of biomedical optics 10(1), P.14010 (2005).

[12] Bekshaev, A.,Y., Angelsky, O., V., Hanson, S., G., and Zenkova, C., Y., "Scattering of inhomogeneous circularly polarized optical field and mechanical manifestation of the internal energy flows," Phys. Rev. A. 86, 023847 (2012).

[13] Angelsky, O.,V., Polyanskii, P.,V., Felde, C.,V., "The emerging field of correlation optics," Optics and Photonics News 23(4), p.p.25-29 (2012).

[14] Ushenko, Y., A., Dubolazov, A., V., Angelsky, A., P., Sidor, M., I., Bodnar, G., B., Koval, G., Zabolotna, N. I., Smolarz, A., Junisbekov, M., Sh., "Laser polarization fluorescence of the networks of optically anisotropic biological crystals," Proc. SPIE (Optical Fibers and Their Applications), 869809 (2012).

[15] Angelsky, P., O., Ushenko, A., G., Dubolazov, A., V., Sidor, M., I., Bodnar, G., B., Koval, G., and Trifonyuk, L., "The singular approach for processing polarization-inhomogeneous laser images of blood plasma layers," J. Opt. 15, 044030 (2013).

[16] Angelsky, O.,V., Yermolenko, S.,B., Zenkova, C.,Yu., Angelskaya, A.,O., "Polarization manifestations of correlation (intrinsic coherence) of optical fields." Applied optics 47 (29), 5492-5499 (2008)

[17] Angelsky, O., Mokhun, A., Mokhun, I., Soskin, M., "The relationship between topological characteristics of component vortices and polarization singularities," Optics communications 207 (1), 57-65 (2002).

[18] Angelsky, O.,V., Burkovets, D.,N., Maksimyak, P.,P., Hanson, S.G., "Applicability of the singular-optics concept for diagnostics of random and fractal rough surfaces," Applied optics 42 (22), 4529-4540 (2003).

[19] Angel'skii, O.,V., Ushenko, O.,G., Burkovets, D.,N., Arkhelyuk, O.,D., Ushenko, Y.,A., "Polarizationcorrelation studies of multifractal structures in biotissues and diagnostics of their pathologic changes." LASER PHYSICS 10(5), 1136-1142 (2000). 